\title{
Matrices Hermitian for an Absolute Norm
}

\author{
HANS SCHNEIDER and ROBERT E. L. TURNER † \\ Department of Mathematics, University of Wisconsin, Madison WI, 53706, U.S.A.
}

(Received Sanuary 6, 1972)

\begin{abstract}
Let $\nu$ be a (standardized) absolute norm on $C_{n}$. A matrix $H$ in $C_{n n}$ is called normHermitian if the numerical range $V(H)$ determined by $v$ is real. Let $\mathscr{Z} \ell$ be the set of all norm-Hermitians in $C_{n n}$. We determine an equivalence relation $\sim$ on $\{1, \ldots, n\}$ with the following property: Let $H \in C_{n n}$. Then $H \in \mathscr{Z}$ if and only if $H$ is Hermitian and $h_{i j}=0$ if $i \neq j$. Let $\mathscr{J}=\mathscr{H}+i \mathscr{X}$. Then $\mathscr{Z}$ is a subalgebra of $C_{n n}$ and, for $A \in \mathscr{Z}$, $V(A)$ equals the Euclidean numerical range and hence is convex. Let $\mathscr{V}$ be the group of isometries for $\nu$, and let $\mathscr{H}=\{\exp (i H): H \in \mathscr{H}\}$. Then $\mathscr{O}$ is a normal subgroup of $\mathscr{V}$ and $\mathscr{V}=\mathscr{U} \mathscr{P}$, where $\mathscr{P}$ is a group of permutation matrices.
\end{abstract}

- For an operator, the concept of the numerical range (field of values) with respect to a norm on the underlying space was introduced independently by Lumer [5] and Bauer [1]. By now there are many interesting applications (cf. Bonsall and Duncan [3]); some of the most fascinating concern normHermitian operators-operators whose numerical range is real. In this paper we consider a special but not unimportant case: (1) Our space will be $C_{n}$, the complex $n$-tuples-concretely given; and (2) We shall consider a norm $v$ which depends only on the absolute values of the coordinates of $x \in C_{n}$. Such norms are called absolute (cf. Bauer, Stoer, Witzgall [2], and Bauer [1]). For the sake of convenience we shall also standardize $v$ so that $v\left(e^{i}\right)=1$, for all canonical basis vectors $e^{i}$ in $C_{n}$.

Our main results are these:

We show that it is possible to determine an equivalence relation $\sim$ on $\{1, \ldots, n\}$ such that a matrix $H$ in $C_{n n}$ is norm-Hermitian if and only iff $H$ is Hermitian, $\ddagger$ and $h_{i j}=0$ if $i \sim j$ (theorem (6.2)). If $\mathscr{H}$ is the set

† The research of one of the authors was supported in part by NSF Grant GP-178:5.

$\ddagger$ We shall always use the term Hermitian matrix $H$ in the traditional sense: $h_{i j}=\overline{h_{j+\infty}}$ $\ell, j=1, \ldots, n$. A matrix with real numerical range will be called norm-Hermitiza y-Hermitian. 
norm-Hermitian nuatrices and $\mathscr{J}=\mathscr{H}+1 \mathscr{H}$, then $\mathscr{J}$ is a subalgebra of $C_{n A}$, and for each $A \in \mathscr{I}$, the numerical range $V(A)$ equals the Euclidean numerical range. Hence $V(A)$ is convex for all $A \in \mathscr{J}$ (theorems (6.3) and (6.4)). Let $v^{0}$ be the operator norm associated with $v$. We also show that $v^{0}(A)=\chi^{0}(A)$, for all $A \in \mathscr{I}$, where $\chi$ is the Euclidean norm on $C_{n}(6.5)$. It is well known that, for $H \in \mathscr{H}$, $\exp (i H)$ is a $v$-isometry on $C_{n}$. By use of out characterization of norm-Hermitian matrices, we show that the set $\mathcal{U}$ of all $v$-isometries of the form $\exp (i H), H \in \mathscr{H}$, forms a normal subgroup of the group $\mathscr{F}$ of all $v$-isometries, and that $\mathscr{V} / \mathscr{U}$ is finite. More precisely, there is a group $\mathscr{P}$ of $y$-isometries which are also permutation matrices such that for cach $V \in \mathscr{Y}$ there exist unique $U \in \mathscr{Z}, P \in \mathscr{P}$ such that $V=U P(7.3,7.7,7.8)$.

While the absoluteness of the norm $v$ plays an essential role in our results, the standardization $v\left(e^{\prime}\right)=1, i=1, \ldots, n$ is a matter of convenience. Thus, a simple modification of our results will make them applicable to all absolute norms. In the case of our main theorems, we give them also in this more general form.

\section{NOTATIONS AND DEFINITIONS}

\subsection{Coordinate subspaces}

Let $C$ be the complex field, $R$ the real field, $R^{+}$the set of nonnegative numbers. We put

$$
C_{n}=\left\{x=\left(x_{1}, \ldots, x_{n}\right): x_{l} \in C\right\}
$$

and defino $R_{n}, R_{\mathrm{n}}^{+}$analogously. By $e^{i}, i=1, \ldots, n$ we denote the vector in $C_{n}$ (or $R_{n}$ ) defined by $e_{i}^{i}=1, e_{j}^{j}=0$ otherwise. We call $e^{l}$ a unit vector. A coordinate subspace of $C_{n}$ (or $R_{n}$ ) is the space spanned by a set of unit vectors,

\subsection{Norms}

On $C_{n}$ (or on any coordinate subspace of $C_{n}$ ), $\chi$ will denote the Euclidean norm

$$
\chi(x)=\left(\left|x_{1}\right|^{2}+\cdots+\left|x_{n}\right|^{2}\right)^{\frac{1}{3}} .
$$

If $x \in C_{n}$, then $|x|=\left(\left|x_{1}\right|, \ldots,\left|x_{n}\right|\right) \in R_{n}^{+}$.

A norm on $C_{n}$ is (as usual) a function v of $C_{n}$ into $R^{+}$such that

i) $v(x)=0$ if and only if $x=0$,

ii) $v(x+z) \leqslant v(x)+v(z)$

iii) $v(\alpha x)=|\alpha| v(x)$, for $\alpha \in C$.

A norm $v$ is called absolute if, in addition, 
iv) $v(x)=v(|x|)$, all $x \in C_{n}$,

and standardized if

v) $v\left(e^{l}\right)=1, \quad i=1, \ldots, n$.

Unless otherwise stated, $v$ will always denote a standardized absolute norm.

We also make the following conventions. If $x \in C_{n}$, then $x_{1}$ is the $i$ th coordinate of $x$. On the other hand, if $C_{n}$ is split as the direct sum of coordinate subspaces: $C_{n}=E_{1} \oplus \cdots \oplus E_{r}, x_{(1)}$ will denote the component of $x$ in $E_{i}$. If $\operatorname{dim} E_{i}=m$, then $x_{(i)} \in C_{m}$, and we therefore write

$$
x=x_{(1)} \oplus \cdots \oplus x_{(r)}
$$

(rather than $\left.x=x_{(1)}+\cdots+x_{(r)}\right)$.

\subsection{Dual norms and numerical ranges of matrices}

For $x, y \in C_{n}$, we put

$$
\langle y, x\rangle=\vec{y}_{1} x_{1}+\cdots+\vec{y}_{n} x_{n} .
$$

If $v$ is a norm on $C_{n}$, so is $v^{D}$ :

$$
v^{D}(y)=\sup _{x \neq 0} \frac{\langle y, x\rangle \mid}{v(x)} .
$$

If $v$ is absolute, so is $v^{D}$ (cf. [2]), and it is easy to see that if $v$ is standardized absolute, so is $v^{D}$.

If $x, y \in C_{n}$, and $1=\langle y, x\rangle=v^{D}(y) v(x)$, then $y$ is called dual to $x$; we write $y \| x$. It is well known that for each $x \in C_{n}, x \neq 0$, there is at least one $y \in C_{n}$ such that $y \| x$, and for each $y \in C_{n}, y \neq 0$, there is an $x \in C_{n}$ such that $y \| x$.

By $C_{n n}$ we denote the set of all $(n \times n)$ matrices over $C$. The numerical range $V(A)$ for $A \in C_{n n}$ is defined by

$$
V(A)=\left\{\langle y, A x\rangle: x, y \in C_{n} \text { and } y \| x\right\} .
$$

If $V(A)$ is real, then $A$ is called norm-Hermitian or $v$-Hermitian.

2

2.1

DEFINITION Let $v$ be a standardized absolute norm $C_{n}$. On $\{1,2, \ldots, n\}$ we define a relation $\sim$ thus: $i \sim j$ if for all $x, y \in C_{n}$ such that

$$
\left|x_{i}\right|^{2}+\left|x_{j}\right|^{2}=\left|y_{i}\right|^{2}+\left|y_{j}\right|^{2} \text {, and }\left|x_{k}\right|=\left|y_{k}\right|, \text { for } k \neq i, j,
$$

we have $v(x)=v(y)$. 


\section{2}

LEMMA The relation $\sim$ is an equivalence relation on $\{1,2, \ldots, n\}$.

Proof Since $v$ is absolute, $i \sim i$ for,$: 1 ;=1,2, \ldots, n$. Clearly, $i \sim j$ implies that $j \sim i$. Suppose that $h, i, j$ are distinct integers with $h \sim i$ and $i \sim j$. For $x \in C_{n}$ define $\tilde{x}$ by $\tilde{x}_{h}=\tilde{x}_{j}=0, \tilde{x}_{l}=\left(\left|x_{h}\right|^{2}+\left|x_{i}\right|^{2}+\left|x_{j}\right|^{2}\right)^{j}$ and $\bar{x}_{k}=\left|x_{k}\right|$ for $k \neq l i, i, j$. It is easy to see that $v(x)=v(\tilde{x})$. Now suppose that $x, y \in C_{n}$, and $\left|x_{k}\right|^{2}+\left|x_{j}\right|^{2}=\left|y_{k}\right|^{2}+\left|y_{j}\right|^{2}$, and $\left|x_{k}\right|=\left|y_{k}\right|$ for $k \neq h, j$. Then $\left|x_{h}\right|^{2}+\left|x_{i}\right|^{2}+\left|x_{j}\right|^{2}=\left|y_{h}\right|^{2}+\left|y_{j}\right|^{2}+\left|y_{k}\right|^{2}$ whence $\tilde{x}=\tilde{y}$. Thus $v(x)=v(\tilde{x})=v(\tilde{y})=v(y)$. It follows that $h \sim j$.

\section{3}

Lemma Let $v$ be a standardized absolute norm on $C_{n}$. Let $N_{1}, \ldots, N_{r}$ be the equivalence classes in $\{1, \ldots, n\}$ given by $\sim$. Let $E_{k}$ be the coordinate subspace spanned by the vectors $e^{i}$ with $i \in N_{k,}$ and write $x \in C_{n}$ as $x=x_{(1)} \oplus \cdots \oplus x_{(r)}$, where $x_{(k)} \in E_{x_{k}}$. Then there is a standardized absolute norm $\mu$ on $C_{r}$ such that $\nu(x)=\mu\left(\chi\left(x_{(1)}\right), \ldots, \chi\left(x_{(r)}\right)\right)$.

Proof Let us suppose that $N_{1}=\{1, \ldots, s)$ (to save writing). Put $x^{(1)}=0 \oplus x_{(2)} \oplus \cdots \oplus x_{(r)}$. Then

$$
\begin{aligned}
v(x) & =v\left(\sum_{i=1}^{s} x_{i} e^{l}+x^{(1)}\right) \\
& =v\left(\left(\left|x_{1}\right|^{2}+\left|x_{2}\right|^{2}\right)^{ \pm} e^{1}+\sum_{l=3}^{s} x_{i} e^{l}+x^{(1)}\right) \cdots \\
& =v\left(\chi\left(x_{(1)}\right) e^{1}+x^{(1)}\right) .
\end{aligned}
$$

After repetitions of this argument, we have

$$
v(x)=v\left(\chi\left(x_{(1)}\right) e^{\prime \prime}+\cdots+\chi\left(x_{(r)}\right) e^{J_{r}}\right),
$$

where $j_{l} \in N_{b}, i=1, \ldots, r$. So, for $\alpha \in C_{r}$, we define

$$
\mu(\alpha)=\nu\left(\sum_{k=1}^{s} a_{k} e^{I_{k}}\right) \text {. }
$$

Then $v(x)=\mu\left(\chi\left(x_{(1)}\right), \ldots, \chi\left(x_{(r)}\right)\right)$, It is easily verified that $\mu$ is a standardized absolute norm on $C_{r}$.

\section{4}

COROLLARY Let $U \in C_{n n}$ be a unitary matrix such that $u_{i j}=0$ if $i \times j$. Then $U$ is a v+isometry (i.e., $v\left(U_{x}\right)=v(x)$ for all $x \in C_{n}$ ).

Proof We may write 
$U=U_{1} \oplus \cdots \oplus U_{r}$, where $U_{i}$ is a unitary matrix on $E_{l}, i=1, \ldots, r$. Since $\chi\left(U_{t} x_{(i)}\right)=\chi\left(x_{(l)}\right), i=1, \ldots, r$, we have

$$
v(x)=\mu\left(\chi\left(x_{(1)}\right), \ldots, \chi\left(x_{(r)}\right)\right)=\mu\left(\chi\left(U_{1} x_{(1)}\right), \ldots, \chi\left(U_{r} x_{(r)}\right)=v(U x) .\right.
$$

\section{5}

COROLlary If $i \sim j$ for all $i, j \in(1, \ldots, n)$, then $v=\chi$.

Proof In this case $x_{(1)}=x \in C_{n}$, and so by (2.3) and since $\mu$ is standardized, $v(x)=\mu(\chi(x))=\chi(x)$.

\section{3}

In this section we shall explore the geometric significance of the equivalence relation introduced in Section 2. We begin with a simple geometric lemma on real 2-space.

If $K$ is a convex body in $R_{2}$, denote its boundary by $\partial K$, and put $K^{+}=K \cap R_{2}^{+}$.

\section{1}

LEMMA Let $K$ be a convex body in $R_{2}$ such that $0 \in K,(1,0) \in \partial K$ and $(0,1) \in \partial K$. Then there exists a $P=\left(x_{1}, x_{2}\right) \in \partial K$ with $x_{1}>0, x_{2}>0$ such that the perpendicular $l$ to $O P$ through $P$ is a support line to $K$.

Proof For each $\theta, 0 \leqslant \theta \leqslant \pi / 2$, let $r(\theta) \in(\cos \theta, \sin \theta) \in \partial K$. Then $r$ is nonzero and continuous in [0, $\pi / 2]$. Hence $r$ attains its maximum $M$ and its minimum $m$ in that interval, and $0<m \leqslant 1 \leqslant M$.

We consider three cases (which overlap).

Case $1 m=1=M$.

In this case $K^{+}$is a quarter circle. If $P$ is any point on the boundary, the perpendicular $l$ through $P$ is a support line to $K$.

Case $21<M$, say $r\left(\theta_{0}\right)=M$. Clearly $0<\theta_{0}<\pi / 2$. Thus $K^{+}$is contained in the circle center $O$, radius $M$. If $P=r\left(\theta_{0}\right)\left(\cos \theta_{0}, \sin \theta_{0}\right)$, then $l$ is a support line.

Case $3 m<1$, say $r\left(\theta_{1}\right)=m$. Again $0<\theta_{1}<\pi / 2$. Let

$$
P=r\left(\theta_{1}\right)\left(\cos \theta_{1}, \sin \theta_{1}\right),
$$

and $l$ the perpendicular to $O P$ at $P$. We claim that $l$ is the (only) support line to $K$ at $P$. Suppose $l$ is not a support line to $K$ at $P$. Then there exists a support line $l^{\prime}$ at $P$, and $l^{\prime}$ is not perpendicular to $O P$. Since $(1,0) \in K$, 
$(0,1) \in K$, and $|O P|=m<1$, the slope of $I^{\prime}$ is negative. Hence the perpendicular to $l^{\prime}$ from $O$ meets $l^{\prime}$ is a point $Q$ in the first quadrant. Clearly $|O Q|<|O P|$. Since $Q$ is either on the boundary of $K$ or in the exterior of $K$, there is a point $R=r\left(0_{2}\right)\left(\cos O_{2}, \sin \theta_{2}\right), 0<0_{2}<\pi / 2$ on $O Q$ which is on the boundary of $K$. Thus

a contradiction.

$$
r\left(0_{2}\right)=|O R| \leqslant|O Q|<|O P|=m,
$$

The lemma is proved.

\section{2}

COROLLARY Let $\kappa$ be a standardized absolute norm on $C_{2}$. Then there exists an $x \in R_{2}^{+}$, with $x_{1}>0$ and $x_{2}>0$ such that $\langle x, x\rangle^{-1} x \| x$.

Proof Let $K=\left\{x \in R_{2}: k(x) \leqslant 1\right\}$. Then $K$ is convex and satisfies the conditions of (3.1). Let $P=x$, where $x_{1}>0, x_{2}>0$, be a point such that the perpendicular $l$ through $P$ to $O P$ is a support line to $K$. Then for all $z \in R_{2}^{+}$, we have $\langle x, z\rangle \leqslant\langle x, x\rangle k(z)$. Since $\kappa$ is absolute, it follows that $|\langle x, z\rangle| \leqslant\langle x, x\rangle \kappa(z)$ for all $z \in C_{2}$. Hence $\kappa^{D}(x)=\langle x, x\rangle$, and $\langle x, x\rangle^{-1} x \| x$.

\section{3}

Definitions 1) Let $1 \leqslant i, j \leqslant n ; i \neq j$. In the rest of this section, we shall write $E^{\prime}=\operatorname{span}\left\{e^{\prime}, e^{\prime}\right\}, E^{n}=\operatorname{span}\left\{e^{k}: k \neq i, f\right\}$. For $x \in C_{\mathrm{n}}$, we shall put $x=x^{\prime} \oplus x^{\prime \prime}$, where $x^{\prime} \in E^{\prime}, x^{\prime \prime} \in E^{\prime \prime}$. Also $x^{\prime}=\left(x_{1}, x_{1}\right)$, and we shall identify $x^{\prime \prime}$ and $0 \oplus x^{\prime \prime}$, where $0 \in E^{\prime}$.

2) Let $K=\left\{x \in C_{n}: v(x) \leqslant 1\right\}$. If $x^{n} \in E^{\prime \prime}$ and $v\left(x^{n}\right) \leqslant 1$, we put

$$
K_{x^{\prime \prime}}=\left\{x^{\prime} \in E^{\prime}: v\left(x^{\prime} \oplus x^{\prime \prime}\right) \leqslant 1\right\} .
$$

We call $K_{x^{\prime \prime}}$ a section of $K$. Suppose that $x^{n} \in E^{\prime \prime}$ and $v\left(x^{\prime \prime}\right) \leqslant 1$. Let $\kappa_{x^{*}}$ be the mapping of $E^{\prime}$ into $R^{+} \mathrm{U}\{\infty\}$ defined by

$$
\kappa_{x^{\prime \prime}}\left(x^{\prime}\right)=\inf \left(\alpha>0: \frac{1}{\alpha} x^{\prime} \in K_{x^{\prime}}\right) \text {. }
$$

(Thus $\kappa_{x^{n}}\left(x^{\prime}\right)=\infty$ if $\beta x^{\prime} \in K_{x^{m}}$ and $\beta \geqslant 0$ imply that $\beta=0$.)

3) I'c shall call $K_{x^{\prime \prime}}$ circular if there is a nonnegatine r such that

$$
K_{x^{4}}=\left\{\left(x_{f}, x_{j}\right):\left|x_{i}\right|^{2}+\left|x_{j}\right|^{2} \leqslant r^{2}\right\} .
$$

4) Let $x=x^{\prime} \oplus x^{\prime}, y=y^{\prime} \oplus y^{\prime \prime}$ be elements of $C_{n^{\prime}}$. We shall write $y \mid x$ if

a) $y \| x$,

and

b) There isia positlte d such that $y^{\prime}=d x^{\prime}$. 


\section{4}

Lemma Let $x^{\prime \prime} \in E^{\prime \prime}$ with $v\left(x^{\prime \prime}\right) \leqslant 1$. Then

1) $K_{x^{\prime \prime}}$ is a convex body in $E^{\prime}$ with $0 \in K_{x^{\prime \prime}}$

2) $x^{\prime} \in K_{x^{\prime \prime}}$ if and only if $\left|x^{\prime}\right| \in K_{x^{\prime \prime}}$.

3) If $v\left(x^{\prime \prime}\right)<1$, then $0 \in$ int $K_{x^{\prime \prime}}$.

4) If $v\left(x^{\prime \prime}\right)<1$, then $\kappa_{x^{\prime \prime}}$ is an absolute norm on $E^{\prime}$ and is standardized if $x^{n}=0$.

Proof 1) Clearly $0 \in K_{x^{\prime \prime}}$ since $v\left(0 \oplus x^{\prime \prime}\right) \leqslant 1$. If $x^{\prime}, y^{\prime} \in K_{x^{\prime \prime}}$ and $0 \leqslant \alpha \leqslant 1$, then

$$
v\left(\alpha x^{\prime}+(1-\alpha) y^{\prime} \oplus x^{\prime \prime}\right) \leqslant \alpha v\left(x^{\prime} \oplus x^{\prime \prime}\right)+(1-\alpha) v\left(y^{\prime} \oplus x^{\prime \prime}\right) \leqslant 1,
$$

when $\alpha x^{\prime}+(1-\alpha) y^{\prime} \in K_{x^{\prime \prime}}$. Thus $K_{x^{\prime \prime}}$ is convex.

2) Since $\left.v\left(x^{\prime} \oplus x^{\prime \prime}\right)=v\left(\left|x^{\prime}\right| \oplus x^{\prime \prime}\right), 2\right)$ follows.

3) Suppose $v\left(x^{\prime \prime}\right)<1$. Then for all $x^{\prime} \in E^{\prime}$ with $v\left(x^{\prime}\right)<1-v\left(x^{\prime \prime}\right)$, we have $v\left(x^{\prime} \oplus x^{\prime \prime}\right) \leqslant v\left(x^{\prime}\right)+v\left(x^{\prime \prime}\right)<1$, whence $x^{\prime} \in K_{x^{\prime \prime}}$. Hence $0 \in \operatorname{int} K_{x^{\prime \prime}}$.

4) Follows immediately from 1), 2), and 3).

\section{5}

THEOREM Let $v$ be a standardized absolute norm on $C_{n}$, and let $1 \leqslant i, j \leqslant n$. Then $i \sim j$ if and only if for all $x^{\prime \prime} \in E^{\prime \prime}$ with $v\left(x^{\prime \prime}\right) \leqslant 1$, the section $K_{x^{\prime \prime}}$ is circular.

Proof Suppose that $i \sim j$ and let $x^{\prime \prime} \in E^{\prime \prime}$ with $v\left(x^{\prime \prime}\right) \leqslant 1$. Let $x^{\prime}, y^{\prime} \in E^{\prime}$ and assume that $\chi\left(x^{\prime}\right)=\chi\left(y^{\prime}\right)$. Then $v\left(y^{\prime} \oplus x^{\prime \prime}\right)=v\left(x^{\prime} \oplus x^{\prime \prime}\right)$. Hence $x^{\prime} \in K_{x^{*}}$ if and only if $y^{\prime} \in K_{x^{\prime \prime}}$. Thus $K_{x^{m}}$ is circular.

Conversely, suppose that $K_{x^{\prime \prime}}$ is circular for all $x^{\prime \prime} \in E^{n}$ with $v\left(x^{\prime \prime}\right) \leqslant 1$. Let $x, y \in E$ and assume that $x=x^{\prime} \oplus x^{\prime \prime}, y=y^{\prime} \oplus y^{\prime \prime}$ where $\chi\left(x^{\prime}\right)=\chi\left(y^{\prime}\right)$ and $\left|x^{\prime \prime}\right|=\left|y^{\prime \prime}\right|$. If $x^{\prime}=0$, then $y^{\prime}=0$ or $v(x)=v(y)$. So suppose that $x^{\prime} \neq 0$. Thus $y^{\prime} \neq 0$. Put $u=x / v(x), v=y / v(x)$ and observe that $v(u)=1$. If $u=u^{\prime} \oplus u^{\prime \prime}$, then $v\left(u^{\prime \prime}\right) \leqslant 1$, since $v$ is absolute (cf. [2]). Thus $u^{\prime} \in K_{u^{\prime \prime}}$. But if $v=v^{\prime} \oplus v^{\prime \prime}$, then $\chi\left(v^{\prime}\right)=\chi\left(u^{\prime}\right)$, and since $K_{u^{\prime}}$ is circular, we also have that $v^{\prime} \in K_{u^{\prime \prime}}$. Further $\left|v^{\prime \prime}\right|=\left|u^{\prime \prime}\right|$, whence $v(v)=v\left(v^{\prime} \oplus u^{\prime \prime}\right) \leqslant 1$. It follows that $v(x) \geqslant v(y)$. Reversing the roles of $x$ and $y$, we oblain $v(y) \geqslant v(x)$, whence $v(x)=v(y)$. Thus $i \sim j$.

\section{6}

LEMMA If for all $x^{\prime \prime} \in E^{n}$ with $v\left(x^{\prime \prime}\right)<1$, the section $K_{x^{\prime \prime}}$ is circular then $K_{x^{\prime \prime}}$ is also circular if $x^{\prime \prime} \in E^{\prime \prime}$ and $v\left(x^{\prime \prime}\right)=1$. 
Proof Let $x^{\prime \prime} \in E^{x}$ with $y\left(x^{\prime \prime}\right)=1$. Let $x^{\prime}, y^{\prime} \in E^{\prime}, x^{\prime} \in K_{x^{\prime \prime}}$ and $\chi\left(y^{\prime}\right)=\chi\left(x^{\prime}\right)$. We must show that $y^{\prime} \in K_{x^{\prime \prime}}$.

Let $0<\varepsilon<1$. Since $y\left(x^{\prime} \oplus x^{\prime \prime}\right) \leqslant 1$ and $y$ is absolute, $y\left(x^{\prime} \oplus(1-\varepsilon) x^{\prime \prime}\right) \leqslant 1$. But $K_{(1-\imath) x^{\prime \prime}}$ is circular, whence $v\left(y^{\prime} \oplus(1-\varepsilon) x^{\prime \prime}\right) \leqslant 1$. Hence also

$$
y\left(y^{\prime} \oplus x^{\prime \prime}\right) \leqslant 1,
$$

and the desired result $y^{\prime} \in K_{x^{\prime \prime}}$ follows.

\section{7}

LEMMA Let $x=x^{\prime} \oplus x^{n} \in R_{n}^{+}$, where $v\left(x^{n}\right)<v(x)=1$. If $y=y^{\prime} \oplus y^{\prime \prime} \in R_{n}^{+}$ and $y \| x$ then $\left.x^{\prime} \neq 0, c^{-1}=1-\left\langle y^{\prime \prime}, x^{n}\right\rangle\right\rangle 0$ and $c y^{\prime} \| x^{\prime}$ with respect to the norm $\kappa_{x^{*}}$.

Proof Clearly $x^{\prime} \neq 0$. Since $v^{D}\left(y^{\prime \prime}\right) \leqslant v^{D}(y) \leqslant 1,1-\left\langle y^{\prime \prime}, x^{\prime \prime}\right\rangle>0$. Hence $\left.c=\left(1-\left\langle y^{\prime \prime}, x^{\prime \prime}\right\rangle\right)^{-1}\right\rangle 0$. Clearly $\left\langle y^{\prime}, x^{\prime}\right\rangle=1-\left\langle y^{\prime \prime}, x^{\prime \prime}\right\rangle$. Let $\kappa_{x^{*}}\left(z^{\prime}\right)=1$. Then also $\kappa_{x^{n}}\left(\left|z^{\prime}\right|\right)=1$, whence $v\left(\left|z^{\prime}\right| \oplus x^{\prime \prime}\right)=1$. Hence

whence

$$
\left\langle y^{\prime},\left|z^{\prime}\right|\right\rangle+\left\langle y^{\prime \prime}, x^{\prime \prime}\right\rangle \leqslant 1
$$

$$
\left|\left\langle y^{\prime}, z^{\prime}\right\rangle\right| \leqslant\left\langle y^{\prime},\left|z^{\prime}\right|\right\rangle \leqslant 1-\left\langle y^{\prime \prime}, x^{n}\right\rangle
$$

Hence $\left(\kappa_{x^{\prime \prime}}\right)^{D}\left(y^{\prime}\right)=1-\left\langle y^{n}, x^{a}\right\rangle=c^{-1}$ and $c y^{\prime} \| x^{\prime}$, with respect to $\kappa_{x^{* *}}$

\section{8}

LEMMA Let $x^{\prime \prime} \in E^{\prime \prime} \cap R_{n-2}^{+}$, where $v\left(x^{n}\right)<1$. Suppose that for all $x^{\prime} \in E^{\prime} \cap R_{2}^{+}$ such that $v(x)=1, x=x^{\prime} \oplus x^{n}$, there is a $y \in R_{n}^{+}$such that $y \mid x$. Then the the section $K_{x}$ is circular.

Proof Let $x^{\prime}$ satisfy the hypotheses of the lemma. Let $y \in R_{t}^{+}, y \| x$, $y^{D}(y)=1$ and $y^{\prime}=d x^{\prime}$, where $d>0$. By (3.7) there is a positive $c$ such that $c y^{\prime} \| x^{\prime}$ and hence $c d x^{\prime} \| x^{\prime}$ with respect to the norm $\kappa_{x^{\prime \prime}}$. Applying Lemma (3.1) of Gries [5], we see that the corresponding norm body $K_{x^{\prime \prime}} \cap R_{2}$ is circular. But by (2) of (3.4), it now follows that $K_{x^{*}}$ is circular in the complex space $E^{\prime}$.

\section{9}

THROREM Let $v$ be a standardized absolute norm and let $E^{\prime}, E^{\prime \prime}$ be defined as in (3.3). Suppose for all $x \in R_{n}^{+}$with $v\left(x^{n}\right)<v(x)=1$ there is a $y \in R_{n}^{+}$such that $y \mid x$. Then $i \sim j$.

Proof Let $x^{0} \in E^{n} \cap R_{n-2}^{+}$, where $v\left(x^{*}\right)<1$, By (3.8), $K_{x^{*}}$ is circular. It follows from (2) of (3.4) that $K_{x^{*}}$ is circular if $x^{n} \in E^{n}$ and $y\left(x^{*}\right)<1$. But now it follows from (3.6) that $K_{x^{n}}$ is circular for all $x^{n} \in E^{n}$ such that $v\left(x^{n}\right) \leqslant 1$, By $(3.5), i \sim j$. 
4.1

LEMMA Let $E_{l}, i=1, \ldots, r$ be coordinate subspaces of $C_{n}$ such that $C_{n}=E_{1} \oplus \cdots \oplus E_{r}$. Let $\lambda_{l}$ be a standardized absolute norm on $E_{l}$, let $\mu$ be a standardized absolute norm on $C_{r}$, and suppose

$$
v\left(x_{(1)} \oplus \cdots \oplus x_{(r)}\right)=\mu\left(\lambda_{1}\left(x_{(1)}\right), \ldots, \lambda_{r}\left(x_{(r)}\right)\right)
$$

where $x_{(i)} \in E_{l}, i=1, \ldots, r$. Then

1) $v$ is a standardized absolute norm on $C_{n}$,

2) $\nu^{D}\left(y_{(1)} \oplus \cdots \oplus y_{(r)}\right)=\mu^{D}\left(\lambda_{1}^{D}\left(y_{(1)}\right), \ldots, \lambda_{r}^{D}\left(y_{(r)}\right)\right)$

for $y_{(i)} \in E_{i}, i=1, \ldots, r$.

Further, let $x=x_{(1)} \oplus \cdots \oplus x_{(r)}, x_{(1)} \in E_{l}, y=y_{(1)} \oplus \cdots \oplus y_{(r)}, y_{(1)} \in E_{l,}$ and suppose that $\lambda_{i}\left(x_{(l)}\right)=\alpha_{i}, \lambda_{i}^{P}\left(y_{(l)}\right)=\beta_{i}, i=1, \ldots, j$. Let $\alpha=\left(\alpha_{1}, \ldots, \alpha_{r}\right)$, $\beta=\left(\beta_{1}, \ldots, \beta_{\mathrm{r}}\right)$. Then

3) $y \| x$ with respect to $v$ if and only if

a) $\beta \| \alpha$ with respect to $\mu$, and

b) $\beta_{l}^{-1} y_{(i)} \| \alpha_{i}^{-1} x_{(l)}$ with respect to $\lambda_{l}$, whenever $\beta_{i} \alpha_{l}>0, i=1, \ldots, r$.

Proof 1) Let

Then $\dagger$

$$
\begin{aligned}
& x=x_{(1)} \oplus \cdots \oplus x_{(r)}, \\
& z=z_{(1)} \oplus \cdots \oplus z_{(r)} .
\end{aligned}
$$

$$
\begin{aligned}
v(x+z)= & \mu\left(\lambda_{1}\left(x_{(1)}+z_{(1)}\right), \ldots, \lambda_{r}\left(x_{(r)}+z_{(r)}\right)\right) \\
& \leqslant \mu\left(\lambda_{1}\left(x_{(1)}\right)+\lambda_{1}\left(z_{(1)}\right), \ldots, \lambda_{r}\left(x_{(r)}\right)+\lambda_{r}\left(z_{(r)}\right)\right) \\
& \leqslant \mu\left(\lambda_{1}\left(x_{(1)}\right), \ldots, \lambda_{r}\left(x_{(r)}\right)\right)+\mu\left(\lambda_{1}\left(z_{(1)}\right), \ldots, \lambda_{r}\left(z_{(r)}\right)\right) \\
& =v(x)+v(z) .
\end{aligned}
$$

Here the first inequality follows from the absoluteness of $\mu$. Similarly,

$$
v(\alpha x)=|\alpha| v(x) \text {, and } v(|x|)=v(x) \text {, }
$$

since all of $\mu$ and $\lambda_{1}$ are absolute. Clearly $\nu\left(e^{\lambda}\right)=\lambda_{j}(f)$, for some $j, 1 \leqslant j \leqslant r_{\text {, }}$ and some unit vector $f$ of $E_{j}$, whence $v\left(e^{\dagger}\right)=1$. This proves 1).

2) and 3) Suppose that $\alpha$ and $\beta$ are defined as in the statement of the lemma. Let $y \in C_{n}$. Then for any $x$ with $\nu(x)=1$ we have $\mu(\alpha)=1$ and

$$
\begin{aligned}
|\langle y, x\rangle| & \leqslant\left|\left\langle y_{(1)}, x_{(1)}\right\rangle\right|+\cdots+\left|\left\langle y_{(r)}, x_{(r)}\right\rangle\right| \\
& \leqslant \beta_{1} \alpha_{1}+\cdots+\beta_{r} \alpha_{r} \\
& \leqslant \mu_{(}^{D}(\beta) \mu(\alpha)=\mu^{D}(\beta) .
\end{aligned}
$$

Hence $\nu^{D}(y) \leqslant \mu^{D}(\beta)$.

$\dagger$ This argument also occurs in Ostrowski [13] p. 12, where it is merely assumed that $\mu$ is monotonic in $R_{n}^{+}$. 
Suppose further that $x$ is so chosen that $\beta \| \alpha$ and that for $i=1, \ldots, r$, $\beta_{t}^{-1} y_{(i)} \| \alpha_{l}^{-1} x_{(1)}$, whenever $\beta_{i} \alpha_{l}>0$. Since $\left\langle y_{(i)}, x_{(J)}\right\rangle=0$ whenever $\beta_{i} \alpha_{i}=0$, it follows that

$$
\begin{aligned}
\left\langle y_{r} x\right\rangle & =\left\langle y_{(1)}, x_{(1)}\right\rangle+\cdots+\left\langle y_{(r)}, x_{(r)}\right\rangle \\
& =\beta_{1} \alpha_{1}+\cdots+\beta_{r} \alpha_{r} \\
& =\mu^{D}(\rho) \mu(x)=1 .
\end{aligned}
$$

Hence $\nu^{D}(y)$ is given by 2), and if $x$ satisfies the conditions of 3), then $y \| x$.

We must still prove that for all pairs, $x, y \in C_{n}$ with $y \| x$, the condilions of 3) are satisfied. So suppose that $y \| x$. Then

and

$$
1=\langle y, x\rangle=v^{D}(y) v(x)=\mu^{D}(\rho) \mu(\alpha)
$$

$$
\begin{aligned}
\langle y, x\rangle & =\left\langle y_{(1)}, x_{(1)}\right\rangle+\cdots+\left\langle y_{(r)}, x_{(r)}\right\rangle \\
& \leqslant\left|\left\langle y_{(1)}, x_{(1)}\right\rangle\right|+\cdots+\left|\left\langle y_{(r)}, x_{(r)}\right\rangle\right| \\
& \leqslant \beta_{1} \alpha_{1}+\cdots+\beta_{r} \alpha_{r} \\
& \leqslant \mu^{D}(\beta) \mu(\alpha) .
\end{aligned}
$$

Hence all inequalities in $\left(^{*}\right)$ are equalities, and

Thus $\beta \| \alpha$ follows.

$$
1=\mu^{D}(\beta) \mu(\alpha)=\beta_{1} \alpha_{1}+\cdots+\beta_{r} \alpha_{r}
$$

Finaliy, suppose that $\beta_{1} \alpha_{1}>0$. Since $\left|\left\langle y_{(1)}, x_{(1)}\right\rangle\right| \leqslant \beta_{1} \alpha_{1}$ and since we have equalities in ("), we may deduce that

$$
\left\langle y_{(\mathrm{l})}, x_{(\mathrm{i})}\right\rangle=\left\langle y_{(\mathrm{l})}, x_{(\mathrm{l})}\right\rangle \mid=\beta_{\mathrm{r}} \alpha_{\mathrm{i}}
$$

Hence $1=\left\langle\beta_{l}^{-1} y_{(i)}, \alpha_{i}^{-1} x_{(l)}\right\rangle=\lambda_{l}^{D}\left(\beta_{i}^{-1} y_{(i)}\right) \lambda_{i}\left(\alpha_{i}^{-1} x_{(l)}\right)$. Thus $\beta_{i}^{-1} y_{(l)} \| \alpha_{i}^{-1} x_{(l)}$.

\section{2}

Counterexample If we drop the condition that the $\lambda_{\text {l }}$ are absolute, then $v$ will still be a norm on $C_{n}$. But the condition that $\mu$ is absolute cannot be omitted. Consider the following counterexample. Let $E_{1}, E_{2}$ be the two onedimensional coordinate subspaces of $C_{2}$. Let $\lambda_{1}\left(x_{1}\right)=\left|x_{1}\right|, \lambda_{2}\left(x_{2}\right)=\left|x_{2}\right|$, and let $\mu\left(\alpha_{1}, \alpha_{2}\right)=\max \left\{\left|\alpha_{1}-\alpha_{2}\right|,\left|\alpha_{2}\right|\right\}$, and let $v\left(x_{1}, x_{2}\right)=\mu\left(\lambda_{1}\left(x_{1}\right), \lambda_{2}\left(x_{2}\right)\right)$. If $x=(2,1), z=(1,-1)$, then $x+z=(3,0)$. Hence

$$
\begin{aligned}
& v(x)=\mu(2,1)=1, \\
& v(z)=\mu(1,1)=1,
\end{aligned}
$$

but

$$
v(x+z)=\mu(3,0)=3 \text {. }
$$

Thus $v(x+z)>v(x)+v(z)$.

We next slightly extend an important result due to Zenger† [12], (2.26).

† See also Sloer and Witzgal] [14], Theorem I. 


\section{3}

LEMMA Let $\mu$ be an absolute norm on $C_{r}$. Let $\gamma_{1} \geqslant 0, \gamma_{1}+\cdots+\gamma_{r}=1$. Then there exist $\alpha, \beta \in C_{r}$, such that $\beta \| \alpha$ and $\beta_{1} \alpha_{i}=\gamma_{i}$.

Proof If all $\gamma_{1}>0$, then the existence of such $\alpha, \beta$ is guaranteed by Zenger's Lemma [12]. So suppose that, after reordering coordinates, $\gamma_{1}>0$, $i=1, \ldots, s, \gamma_{l}=0, i=s+1, \ldots, r$, where $s<r$. There exist $\alpha^{\prime}, \beta^{\prime} \in C_{s}$ such that $\beta^{\prime} \| \alpha^{\prime}$ (with respect to the restriction of $\mu$ to $C_{s}$ ), and $\beta_{i}^{\prime} \alpha_{i}^{\prime}=\gamma_{l}$, $i=1, \ldots, r$. Let $\beta=\beta^{\prime} \oplus 0, \alpha=\alpha^{\prime} \oplus 0$, where 0 is zero vector of $C_{r-s}$. Since $\mu$ is absolute, $\beta \| \alpha$ and clearly $\beta_{l} \alpha_{l}=\gamma_{l}, i=1, \ldots, r$.

Remark Since $\beta \| \alpha$ implies that $\lambda^{-1} \beta \| \lambda \alpha$, for $\lambda>0$, we may normalize $\mu(\alpha)=\mu^{D}(\beta)=1$, in the above lemma.

\section{4}

DeFinition Let $\Sigma_{1}, \ldots, \Sigma_{r}$ be subsets of the complex plane. We define theconvex sum of $\Sigma_{1}, \ldots, \Sigma_{r}$ to be the set of all sums $\alpha_{1} \sigma_{1}+\cdots+\alpha_{r} \sigma_{r}$, where $\sigma_{i} \in \Sigma_{i}, 0 \leqslant \alpha_{i} \leqslant 1, i=1, \ldots, r$ and $\sum_{i=1}^{r} \alpha_{i}=1$.

Observe that the convex sum of sets need not be a convex set.

\section{5}

LEMMA Let $E_{1}, \ldots, E_{r}$ be coordinate subspaces of $C_{n}$, and let $v$ be given as in (4.1). Let $A=A_{1} \oplus \cdots \oplus A_{r}$, where $A_{i}$ is a matrix acting on $E_{i}, i=1, \ldots, r$. Then the numerical range of $A$ is the convex sum of $V_{1}\left(A_{1}\right), \ldots, V_{r}\left(A_{r}\right)$, where $V_{1}\left(A_{1}\right)$ is the numerical range of $A_{1}$ with respect to the norm $\lambda_{l}$.

Proof Let $\gamma \in R_{r}^{+}, \sigma_{i} \in V_{i}\left(A_{i}\right), i=1, \ldots, n$ and $\sigma=\sum_{i=1}^{r} \gamma_{i} \sigma_{i}$ where $\sum_{i=1}^{r} \gamma_{i}=1$.

Then there exist $y_{(i)}, x_{(l)} \in E_{l}$, such that $y_{l} \| x_{l}$ with respect to $\lambda_{l}$,

$$
\lambda_{i}\left(x_{i}\right)=\lambda \lambda_{i}\left(y_{i}\right)=1 \text {, and }\left\langle y_{(i)}, A_{i} x_{(i)}\right\rangle=\sigma_{i} .
$$

By (4.3) there exists $\alpha, \beta \in R_{1}^{+}$such that $\beta \| \alpha$ with respect to $\mu$, and $\beta_{t} \alpha_{1}=\gamma_{1}$. Let

$$
\begin{aligned}
& x=\alpha_{1} x_{(1)} \oplus \cdots \oplus \alpha_{r} x_{(r)}, \\
& y=\beta_{1} y_{(1)} \oplus \cdots \oplus \beta_{r} y_{(r)} .
\end{aligned}
$$

By (4.1), $y \| x$ with respect to $v$. But

$$
\langle y, A x\rangle=\sum_{i=1}^{r} \beta_{i} \alpha_{i}\left\langle y_{(1)}, x_{(1)}\right\rangle .
$$




$$
=\sum_{i=1}^{r} \lambda_{i} \sigma_{l}=\sigma
$$

whence $\sigma \in V(A)$.

Conversely, let $\sigma \in V(A)$, say $\sigma=\langle y, A x\rangle$ where $y \| x$ with respect to $v$. Let us now write

$$
\begin{aligned}
& x=x_{(1)}^{\prime} \oplus \cdots \oplus x_{(r)}^{\prime} \\
& y=y_{(1)}^{\prime} \oplus \cdots \oplus y_{(r)}^{\prime},
\end{aligned}
$$

where we put $\alpha_{i}=\lambda_{i}\left(x_{(0)}^{\prime}\right), \beta_{i}=\lambda_{i}^{P}\left(y_{(0)}^{\prime}\right)$. Let us suppose that $\gamma_{i}=\beta_{l} \alpha_{i}>0$, $i=1, \ldots, s \leqslant r$, and $\gamma_{l}=\beta_{i} \alpha_{l}=0, t=s+1, \ldots, r$. Then putting $x_{(i)}=\alpha_{l}^{-1} x_{(j)}^{\prime}, y_{i}=\beta_{(0)}^{-1} y^{\prime}, i=1, \ldots, s$, we have by (5.1) that $\beta \| \alpha$ with respect to $\mu$, and $y_{(i)} \| x_{(1)}$ with respect to $\lambda_{l}, i=1, \ldots, s$. Hence

$$
\sigma_{i}=\left\langle y_{(i)}, A_{1} x_{(i)}\right\rangle \in V_{1}\left(A_{i}\right), \quad i=1, \ldots, s .
$$

But $y_{i} \geqslant 0$ and $\sum_{i=1}^{r} y_{i}=1$, and so

$$
\langle y, A x\rangle=\sum_{i=1}^{r}\left\langle y_{(l)}, A_{i} x_{(l)}\right\rangle=\sum_{i=1}^{s} \gamma_{i} \sigma_{i}=\sum_{i=1}^{r} \gamma_{i} \sigma_{i}
$$

The lemma is proved.

Comment Thus, for a norm $v$ satisfying $v(x)=\mu\left(\lambda_{1}\left(x_{(1)}\right), \ldots, \lambda_{r}\left(x_{(r)}\right)\right)$, as in (4.1.1), and $A=A_{1} \oplus \cdots \oplus A_{r}$, the numerical range $V(A)$ does not depend on $\mu$. In particular, if $v$ is any (standardized) absolute norm on $C_{n}$, and $D=\operatorname{diag}\left(d_{1}, \ldots, d_{n}\right)$, then $V(D)$ is the convex hull of $d_{1}, \ldots, d_{n}$, ef. Gries [5].

If $v$ is any norm on $C_{n}$, then the corresponding operator norm $v^{0}$ on $C_{n}$ is defined

$$
v^{0}(A)=\sup \{v(A x): v(x)=1\} .
$$

It is well known, and easy to prove, that

$$
v^{0}(A)=\sup \left\{|\langle y, A x\rangle|: v(x)=1, v_{1 .}^{D}(y)=1\right\} .
$$

\section{6}

LEMMA Let $E_{l}, i=1, \ldots, r$ be coordinate stibspaces of $\mid V$ such that $E_{1} \oplus \cdots \oplus E_{r}=C_{n}$. Let $\lambda_{l}$ be a standardized absolute norm on $E_{l}, i=1, \ldots, r$ and $\mu$ a standardized absolute norm on $C_{r}$, and let $v$ be given by (4.1.1). Let $A \in C_{n n}$ and 'suppose 'that $A=A_{1} \oplus \cdots \oplus A_{r}$, where $A_{l}$ is a matrix on $E_{l^{*}}$ Then

$$
v^{0}(A)=\max \left\{\lambda_{l}^{0}\left(A_{1}\right): \quad l=1, \ldots, r\right\} .
$$

Proof Let $\max \left\{\lambda_{l}^{0}(A): i=1, \ldots, r\right\}=\lambda_{k}^{0}\left(A_{k}\right)$, where $1 \leqslant k \leqslant n$. Then using (4.1), we obtain 


$$
\begin{aligned}
v^{0}(A) & =\sup \left\{|\langle y, A x\rangle|: v(x)=1, v^{D}(y)=1\right\} \\
& \leqslant \sup \left\{\sum_{l=1}^{r}\left|\left\langle y_{(i)}, A_{l} x_{(l)}\right\rangle\right|: \lambda_{l}\left(x_{(i)}\right)=\alpha_{l}, \lambda_{l}^{D}\left(y_{(i)}\right)=\beta_{l}, \mu(\alpha)=\mu^{D}(\beta)=1\right\} \\
& \leqslant \sup \left\{\sum_{i=1}^{r}\left|\beta_{i} \lambda_{l}^{0}\left(A_{i}\right) \alpha_{i}\right|: \alpha, \beta \in E_{n}^{+}, \mu(\alpha)=\mu^{D}(\beta)=1\right\} \\
& \leqslant \lambda_{k}^{0}\left(A_{k}\right)\left(\sum_{i=1}^{r} \beta_{i} \alpha_{i}: \alpha, \beta \in R_{r}^{+}, \mu(\alpha)=\mu^{D}(\beta)=1\right) \\
& \leqslant \lambda_{k}^{0}\left(A_{k}\right) .
\end{aligned}
$$

On the other hand, let $x_{(k)}, y_{(k)} \in E_{k}$ such that $\lambda_{k}\left(x_{(k)}\right)=\lambda_{k}^{D}\left(y_{(k)}\right)=1$, and $\lambda_{k}^{0}\left(A_{k}\right)=\left\langle y_{(k)}, A_{k} x_{(k)}\right\rangle$. If $x_{(i)}=y_{(l)}=0$, for $i \neq k$, then $v(x)=\mu\left(e^{k}\right)=1$, $v^{D}(y)=\mu^{D}\left(e^{k}\right)=1$, and $\langle y, A x\rangle=\left\langle y_{(k)}, A_{k} x_{(k)}\right\rangle=\lambda_{k}^{0}\left(A_{k}\right)$.

The lemma is proved.

We comment that it is almost as easy to prove (4.6) directly from the definition $v^{0}(x)=\sup \{v(A x): v(x)=1\}$, without use of (4.1). When $\operatorname{dim} E_{i}=1, i=1, \ldots, n,(4.6)$ reduces to the well known theorem that $v^{\circ}(D)=\max \left\{\left|d_{i l}\right|, i=1, \ldots, n\right\}$ for a diagonal matrix $D$, cf. [2].

5

\section{1}

LEMMA Let $\Omega=\left\{u \in C_{n}:\left|u_{i}\right|=1\right\}$. Let $K \in C_{n n}$ be a Hermitian matrix such that $k_{i i}=0, i=1, \ldots, n$. If $\langle u, K u\rangle=0$ for all $u \in \Omega$, then $K=0$.

Proof The proof is by induction on $n$. Evidently the result is true for $n=1$. Suppose that it holds for $n=r-1$, and let $n=r$.

Setting $u_{i}=e^{i \theta_{i}}$, we have

whence

$$
\begin{aligned}
\langle u, K u\rangle & =\sum_{1 \leqslant i, j \leqslant r} k_{i j} e^{-i\left(\theta_{j}-\theta_{i}\right)} \\
& =2 \sum_{1 \leqslant i \leqslant j \leqslant r} \operatorname{Re}\left(k_{i j} e^{i\left(\theta_{1}-\theta_{j}\right)}\right.
\end{aligned}
$$

$$
-\operatorname{Re}\left(\left(\sum_{1 \leqslant i \leqslant r-1} k_{l r} e^{-i \theta_{l}}\right) e^{i \theta_{r}}\right)=\operatorname{Re}\left(\sum_{1 \leqslant i<j<r-1} k_{i j} e^{-i\left(\theta_{t}-\theta_{j}\right)}\right)
$$

Since this holds for all $\theta_{r}$, it follows that

$$
\sum_{1 \leqslant i \leqslant r-1} k_{l r} e^{-i \theta_{l}}=0
$$

Again, this holds for all $\theta_{1}, \ldots, \theta_{r-1}$.

We can choose $(r-1)$ linearly independent vectors

$$
\left(e^{-1 \theta}, \ldots, e^{-t \theta(r-1)}\right), \quad \text { e.g., } \quad v^{s}=\left(\omega^{s}, \omega^{2 s}, \ldots, \omega^{(r-1) s}\right)
$$


where $\omega$ is a primitive $r$ th root of 1 . Hence

Now we obtain that for all $\theta_{i}$

$$
k_{r i}=k_{l r}=0, \quad i=1, \ldots, r-1 \text {. }
$$

$$
\sum_{1 \leqslant i, j \leqslant r-1} k_{i j} e^{-\left(\theta_{j}-\theta_{i}\right)}=0
$$

whence by inductive assumption $k_{l j}=0, i, j=1, \ldots, r-1$. Thus $K=0$.

The lemma follows by induction.

\section{2}

LEMmA Let $\Omega=\left\{u \in C_{n}:\left|u_{i}\right|=1, i=1, \ldots, n\right\}$. Let $A \in C_{n n}$, where $a_{\imath}$ is real, $i=1, \ldots, n$. Iffor all $u \in \Omega,\langle u, A u\rangle$ is real, then $A$ is Hermitian.

Proof Let $A=H+i K$, where $H, K$ are Hermitian. Then $k_{u}=0$, $i=1, \ldots, n$. Since $\langle u, K u\rangle=0$, for all $u \in \Omega$, we obtain $K=0$ by (5.1). Hence $A=H$.

6

6.1

LEMMA Let $v$ be a standardized absolute norm. Let $N_{1}, E_{b}$ be as in (2.3). If $A \in C_{n H}$ is such that $A=A_{1} \oplus \cdots \oplus A_{r}$, where $A_{1}$ is a matrix on $E_{l}$, then $V(A)=V_{\mathrm{z}}(A)$, where $V_{\mathrm{x}}(A)$ is the Euclidean numerical rantge.

Proof Let $x=x_{(1)} \oplus \cdots \oplus x_{(r)}$, where $x_{(1)} \in E_{l}$. By (2.3)

$$
v(x)=\mu\left(\chi\left(x_{(1)}\right), \ldots, \chi\left(x_{(r)}\right)\right) \text {, }
$$

where $\mu$ is a standardized absolute norm on $C_{r}$. By (4.5), therefore $V(A)$ is the convex sum of the $V_{l}\left(A_{1}\right), i=1, \ldots, r$. But $V_{i}\left(A_{i}\right)=V_{x}\left(A_{1}\right)$ since $\lambda_{i}=\chi$.

Next, note that

$$
\chi(x)=\grave{\chi}\left(\chi\left(x_{(1)}\right), \ldots, \chi\left(x_{(r)}\right)\right)
$$

and recall the comment after (4.5) that $V(A)$ does not depend on $\mu$. Thus $V_{x}(A)$ is also the convex sum of the $V_{x}\left(A_{1}\right), i=1, \ldots, n$. Thus $V(A)=V_{x}(A)$.

\section{2}

THEOREM Let $v$ be a standardized absolute norm on $C_{n}$, Let the equivalence $\sim$ be defined as in (2.1). Then $H \in C_{N A}$ is norm-Hermitian if and only if
a) $h_{l j}=h_{j 1}$ for $i \sim j$,

and

b) $h_{y j}=0$ for $i \sim j$.

Proof Sưppose $H$ satisfies a) and b). If $N_{b}, E_{\gamma}, i \Rightarrow 1, \ldots, r$ are defincd as 
in (2.3), then $H=H_{1} \oplus \cdots \oplus H_{r}$, where $H_{l}$ is a Hermitian matrix on $E_{i}$. Hence, by $(6.1), V(H)=V_{x}(H)$, which is real. Thus $H$ is norm-Hermitian.

Conversely, suppose that $H$ is norm-Hermitian. Since $e^{t} \| e^{t}, i=1, \ldots, n$, it follows that $h_{u l}=\left\langle e^{l}, H e^{i}\right\rangle$ is real. Suppose that $x, y \in R_{n}^{+}, y \| x$. Let $K \in C_{n n}$ be given by $k_{i j}=y_{l} h_{l j} x_{j}, i, j=1, \ldots, n$. Let $\Omega=\left\{u \in C_{n}:\left|u_{i}\right|=1\right.$, $i=1, \ldots, n\}$. If we define $v, w \in C_{n}$, by $v_{l}=u_{l} x_{i}, w_{i}=u_{l} y_{i}$, then $w \| v$. Hence $\langle w, H v\rangle=\langle u, K u\rangle$ is real. But $k_{b}$ is real, $i=1, \ldots, n$. Hence, by (5.2), $K$ is Hermitian. It follows that

c) $y_{j} h_{j l} x_{i}=y_{i} h_{i j} x_{j}, \quad i, j=1, \ldots, n$ for all $y, x \in R_{n}^{+}$with $y \| x$.

Now let $i, j$ be two fixed integers in $\{1,2, \ldots, n\}$ such that $h_{i j} \neq 0$. We must prove that $\bar{h}_{j l}=h_{t j}$ and that $i \sim j$. We shall use the notation of (3.3). Thus for $x^{\prime} \in E^{\prime}, \kappa_{0}\left(x^{\prime}\right)=v\left(x^{\prime} \oplus 0\right)$, where, by (3.4), $\kappa_{0}$ is a standardized absolute norm on $E^{\prime}$.

Hence, by (3.2) we can find an $x^{\prime} \in E^{\prime} \cap R_{2}^{+}$such that both coordinates of $x^{\prime}$ are positive, $\kappa_{0}\left(x^{\prime}\right)=1$ and there is a positive $c$ for which $c x^{\prime} \| x^{\prime}$ with respect to $\kappa_{0}$. If $x=x^{\prime} \oplus 0 \in C_{n}, y=c x^{\prime} \oplus 0$, then $x_{1}>0, x_{j}>0, y_{1}>0$, $y_{j}>0$. Further, $y \| x$ with respect to $v$, since $\langle y, x\rangle=\left\langle y^{\prime}, x^{\prime}\right\rangle=1$ and for any $z=z^{\prime} \oplus z^{\prime \prime}, v(z)=1$. Since for this particular $x$ and $y$, we have $y_{j} x_{i}=y_{i} x_{j} \neq 0$, it follows from c) that $h_{j l}=h_{i j} \neq 0$.

We may now deduce from $\mathrm{c}$ ) that

d) $y_{j} x_{i}=y_{i} x_{j}$, for all $y, x \in R_{n}^{+}$with $y \| x$.

Suppose that $x \in R_{n}^{+}$and that $v\left(x^{\prime \prime}\right)<v(x)=1$. Since $v$ is absolute, there is a $y \in R_{n}^{+}$such that $y \| x$. Then by d), $y^{\prime}=d_{x} x^{\prime}$ where $d_{x} \geqslant 0$. But by (3.7), $y^{\prime} \neq 0$, whence $d_{x}>0$. Thus $y \mid x$. It now follows by (3.9) that $i \sim j$, and the theorem is proved.

\section{3}

THEOREM Let $v$ be a standardized absolute norm on $C_{n}$. Let

$$
\mathscr{J}=\{H+i K: H, K \text { are norm-Hermitian }\} \text {. }
$$

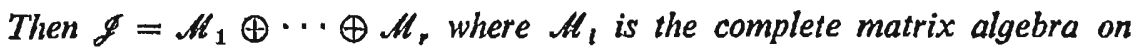
$E_{1}$. Further $\mathscr{J}$ is a subalgebra of $C_{n n}$.

Proof Since any matrix $A_{i}$ on $E_{l}$ is of form $A_{i}=H_{i}+i K_{l}$, where $H_{i}, K_{1}$ are Hermitian, it follows that $A \in \mathscr{J}$ if and only if $A=A_{1} \oplus \cdots \oplus A_{r}$, where $A_{l}$ is a matrix on $E_{l}$. The result follows immediately.

\section{4}

THEOREM If $A \in \mathscr{J}$, then $V(A)=V_{z}(A)$ and is convex.

Proof By (6.3), $A=A_{1} \oplus \cdots \oplus A_{r}$, where $A_{l}$ is a matrix on $E_{l}$. Hence, by $(6.1), V(A)=V_{\mathrm{z}}(A)$, which is convex. 
An important theorem due to Vidav [10] and Palmer [9] (cF. Bonsall and Duncan [3], p. 65) is now stated in a slightly special cuse. Let $V$ be a Banach space and let $\&$ be an algebra of operators on $V$ (normed by the operator norm) such that for eacls $A \in \mathscr{A}, A=H+i K$ where $H, K$ are normHermitian. Define $A^{*}=H-i K$. Then there exists a Hilbert space $V^{\prime}$ and an isomorphism of $A$ onto an algebra of operators $x^{\prime \prime}$ on $V^{\prime}$ which preserves both the norm and the star operation.

Given the dimension of $V$, the Vidav-Palmer theorem by itself gives no information on the dimension of $V^{\prime}$. In our special case, the impact of our next theorem is that one may choose $V^{\prime}=V$.

\section{5}

THEOREM Let $A \in \mathscr{F}$. Then $v^{0}(A)=\chi^{0}(A)$.

Proof By (6.4), $A=A_{1} \oplus \cdots \oplus A_{n}$, where $A_{l}$ is a matrix on $E_{i}$. Hence, by (2.3) and (4.6), $\nu^{0}(A)=\max \left\{\chi^{0}(A): i=1, \ldots, r\right\}$. But

$$
\chi(x)=\chi\left(\chi\left(x_{(1)}\right), \ldots, \chi\left(x_{r}\right)\right)
$$

whence again, by (4.6), $\chi^{0}(A)=\max \left\{\chi^{0}\left(A_{1}\right): i=1, \ldots, r\right\}$. The theorem follows.

There are, of course, many obvious corollaries to (6.2) and (6.5). We shall give some immediate applications to $y$-normal matrices.

\section{6}

Definition $A$ matrix $A \in C_{\mathrm{nn}}$ is called v-normal if $A=H+i K$, where $H, K$ are v-Hermition and $H K=K H$.

If $v=\chi$, a $v$-normal matrix is normal in the traditional sense.

\section{7}

THEOREM Let $v$ be a standardized absolute norm and let $A$ be in $C_{n n}$. Then

1) $A$ is v-nonnal if and only if $A$ is normal and $a_{1}=0$ for $i \sim j$. If $A$ is v-normal, then

2) $V(A)=\operatorname{co}(\operatorname{sp} A)$

3) $v(A)=\rho(A)$.

4) $v^{n}(A)=\rho(A)$.

(Here co(sp $A)$ is the convex hull of the spectrum of $A, v(A)=\sup \{|\lambda|: \lambda \in V(A)\}$, the numerlcal radius of $A$, and $\rho(A)$ is the spectral radius of $A$.)

Proof 1) The matrix $A$ is $v$-normal if and only if $A=H+i K$, where $H, K$ are Fermitian and $h_{i j}=k_{i j}=0$ for $i \sim j$. 
2) By (6.3), $V(A)=V_{\chi}(A)$, and $V_{\chi}(A)=\operatorname{co}(\operatorname{sp} A)$.

3) Immediate.

4) Since $A \in \mathscr{J}, v^{0}(A)=\chi^{0}(A)$, by $(6.6)$ and $\chi^{0}(A)=\rho(A)$.

For operators on a Banach space, 3 ) is known to be true (Palmer [8]), and his proof is much less elementary. By Sinclair's theorem [3, p. 54], $\rho(A)=v^{0}(A)$, where $A$ is a norm-Hermitian operalor. However, Crabb [4] has given a counterexample to 4 ) for a non-absolute norm on $C_{4}$.

7.1

DEFINITIONS Let $v$ be a standardized absolute norm.

i) The set of all norm-Hermitian $H$ in $C_{n n}$ will be denoted by $\mathscr{H}$.

ii) Let $\mathscr{U l}$ be the set of all $U \in C_{n n}$ such that $U=\exp (i H)$ for some $H \in \mathscr{H}$.

iii) The set of all isometries $V \in C_{n n}$ will be denoted by $\mathscr{r}$.

The following theorem is known (cf. Bonsall and Duncan [3, p. 46]) (where it is stated for Banach Aigebras): A matrix $H \in C_{n n}$ is norm-Hermitian, if and only if $\exp (i t H)$ is an isometry for all real $t$. Thus $\mathscr{U} \subseteq \mathscr{V}$. However, our special case is so simple that there is no need to appeal to the above theorem, and our conclusion is stronger than $\mathscr{U} \subseteq \mathscr{V}$. We first state a lemma.

\section{2}

LEMMA Let $v$ be a norm on $C_{n}$. If $V \in \mathscr{V}$, and $H \in \mathscr{H}$, then also $V^{-1} H V \in \mathscr{H}$.

Proof Let $v_{V}$ be defined by $v_{V}(x)=v(V x)$, for all $x$ in $C_{n}$. Since $V \in \mathscr{V}$, $v_{V}=v$. Let $y \| x$, and put $v=V x, w=\left(V^{-1}\right)^{*} y$. It follows from Lemma 1 of [7] that $w \| v$. Hence $\left\langle y, V^{-1} H V x\right\rangle=\langle w, H v\rangle$ is real.

Remark If $v \neq \chi$, then there exists a nonsingular $Z$ such that $Z^{-1} \mathscr{H} Z=\mathscr{H}$ where $Z$ is not a scalar multiple of an isometry. For then we have $r$ classes $N_{1}, \ldots, N_{r}$ for the equivalence relation $\sim$, where $r \geqslant 2(2.5)$. Let $I_{i}$ be the identity on $E_{i}$, and put $Z=\alpha_{1} I_{1} \oplus \cdots \oplus \alpha_{r} I_{r}$, where $\alpha_{i}>0, i=1, \ldots, r$ and $\alpha_{1} \neq \alpha_{r}$. If $v=\chi$, then $Z^{-1} \mathscr{H} Z=\mathscr{H}$ implies that $Z$ is a scalar multiple of a unitary matrix. A simple proof uses the factorization $Z=U D V$, where $U, V$ are unitary and $D=\operatorname{diag}\left(d_{1}, \ldots, d_{n}\right), d_{i}>0$, (essentially) the polar decomposition of $Z$.

\section{3}

THeOREM Let $v$ be a standardized absolute norm on $C_{n}$. Let $\mathscr{V}$ and $\mathscr{U}$ be defined as in (7.1). 
1) A matrix $U \in \mathscr{U}$ if and only if $U$ is unitary and $u_{t j}=0$ for $i * j$.

2) $\mathscr{W}$ is a group and $\mathscr{k}$ is a normal subgroup of $\mathscr{W}$.

Proof 1) Let $\mathscr{H}$ be the set of all norm-Hermitian matrices. Define $E_{k}, k=1, \ldots, r$ as usual, and let $\mathscr{H}_{k}$ be the set of (traditional) Hermitian matrices on $E_{k}$. Then, by Theorem (6.2), $\mathscr{H}=\mathscr{H}_{1} \oplus \cdots \oplus \mathscr{H}_{r}$. Thus $U \in \mathscr{U}$ if and only if $U=U_{1} \oplus \cdots \oplus U_{\mathrm{r}}$, where $U_{l}=\exp \left(i H_{l}\right), H_{l} \in \mathscr{H}_{1}$. But $\exp \left(i \mathscr{H}_{r}\right)$ is well known (and easily seen) to be the set of all unitary matrices $\mathscr{U}_{l}$ on $E_{1}$. Hence $\mathscr{U}=\mathscr{U}_{1} \oplus \cdots \oplus \mathscr{U}_{r}$, which is the assertion 1).

2) Since $\mathscr{U}_{k}$ is the group of all unitary matrices on $E_{k}, k=1, \ldots, r$, it follows from 1): $\mathscr{U}=\mathscr{U}_{1} \oplus \cdots \oplus \mathscr{U}_{\text {r }}$, that $\mathscr{U}$ is a group. By Lemma (2.4), $\mathscr{U} \subseteq \mathscr{r}$.

If $V_{1}, V_{2} \in \mathscr{r}$, so is $V_{1} V_{2}^{-1}$, whence $\mathscr{V}$ is a subgroup of the group of nonsingular matrices.

Let $V \in \mathscr{V}, U \in \mathscr{U}$, say $U=\exp (i H)$, with $H \in \mathscr{H}$. By (7.2), $V^{-1} H V \in \mathscr{H}$, and $\exp \left(i V^{-1} H V\right)=V^{-1} \exp (i H) V=V^{-1} U V$. Thus $V^{-1} U V \in \mathscr{U}$, and so $\mathscr{U}$ is a normal subgroup of $\mathscr{V}$.

Remark For an arbitrary norm $v$ on $C_{m}$ we do not know if $\mathscr{Q}$ is a group.

\section{4}

DEFINITIONS AND REMARKS 1) Let $\left\{N_{1}, \ldots, N_{r}\right\}$ be the equivalence classes for $\sim$ in $\{1,2, \ldots, n\}$.

Denote the symmetric group on $\{1, \ldots, n\}$ by $S_{n}$. Let $\pi$ be a permutation in $S_{n}$, We call $\pi$ a block permutation if

a) For each $k, k=1, \ldots, r$ there is an l such that $\pi\left(N_{k}\right)=N_{\text {, }}$,

b) If $l, j \in N_{k}$ and $i<j$, then $\pi(i)<\pi(j)$, for $k=1, \ldots, r$.

2) If $\pi \in S_{n}$ is a block permutalion, then there is a unique permutation $\rho \in S_{r}$ such that $\pi\left(N_{k}\right)=N_{\rho(k)}, k=1, \ldots, r$. Further, $\left|N_{p(k)}\right|=\left|N_{k}\right|$ where $\left|N_{k}\right|$ is the number of elements in $N_{k}$.

3) If $\pi \in S_{\pi}$, let $P_{n}$ be the permutation matrix defined by $P_{n} e^{\prime}=e^{\pi(n)}$, $i=1, \ldots, n$. If $\pi$ is a block permutation, then $P_{\mathrm{n}}$ will be called $a$ block permutation matrix.

4) The set of all block permutation matrices form a group $Q$ under multiplication.

5) A block permutation which is also an isometry will be called a block isometry.

6) The set of block isometries form a subgroup $\mathscr{P}$ of $Q$ under multiplicallon.

7) It is easy to prove that $|Q|=\prod_{i=1}^{n} t_{1} \leqslant r$ ! where $t_{i}$ is the maximum of 1 and the number of $N_{k}$ with $\left|N_{k}\right|=i$. 
LeMma Let $v$ be an absolute norm, and let $P=P_{\sigma}$ be a permutation matrix which is also an isometry. If $i \sim j$, then $\sigma(i) \sim \sigma(j)$.

Proof Let $E^{\prime}=\operatorname{span}\left\{e^{l}, e^{j}\right\}, E^{\prime \prime}=\operatorname{span}\left\{e^{k}: k \neq i, j\right\}, E_{\sigma}^{\prime}=\operatorname{span}\left\{e^{\sigma(i)}\right.$, $\left.e^{\sigma(J)}\right\}, E_{\sigma}^{\prime \prime}=\operatorname{span}\left\{e^{\sigma(k)}: k \neq i, j\right\}$. Let $x=\sum_{i=1}^{n} x_{l} e^{l}, y=\sum_{i=1}^{n} y_{l} e^{l}$, where $\left|x_{\sigma(i)}\right|^{2}+\left|x_{\sigma(J)}\right|^{2}=\left|y_{\sigma(l)}\right|^{2}+\mid y_{\sigma(j)}{ }^{2}$, and $\left|x_{\sigma(k)}\right|=\left|y_{\sigma(k)}\right|, k \neq i, j$. We wish to prove that $v(x)=v(y)$.

Let $\hat{x}=P^{-1} x, \hat{y}=P^{-1} y$. Then $\hat{x}_{k}=x_{\sigma(k)}, \hat{\theta}_{k}=y_{\sigma(k)}, k=1, \ldots, n$. Hence $\left|\hat{x}_{i}\right|^{2}+\left|\hat{x}_{j}\right|^{2}=\left|\hat{y}_{i}\right|^{2}+\left|\hat{y}_{j}\right|^{2}, \quad\left|\hat{x}_{k}\right|=\left|\hat{y}_{k}\right|, k \neq i, j$. Since $i \sim j$, we have $v(\hat{x})=v(\hat{y})$. But $P$ is an isometry, and so $v(x)=v(y)$.

\section{6}

COROLlaRY If $P_{\sigma}$ is both a permutation matrix and an isometry, then $P_{\sigma}=P_{\rho} P_{\pi}$, where $P_{\rho} \in \mathscr{U}$ and $P_{n} \in \mathscr{P}$.

Proof Let $1 \leqslant k \leqslant r$. By (7.5), there is an $l$ such that $P_{o}\left(E_{k}\right) \subseteq E_{l}$. Hence $\sigma\left(N_{k}\right) \subseteq N_{l}$. But the sets $N_{k}, k=1, \ldots, r$ are finite, and $\sigma$ is $1-1$ and onto $\{1, \ldots, n\}$. Hence there is a permutation $\tau$ in $S_{r}$ such that $\sigma\left(N_{k}\right)=$ $N_{\tau(k)}, k=1, \ldots, r$. Let $\pi$ be the corresponding block permutation in $S_{n}$. Clearly $\sigma\left(N_{k}\right)=\pi\left(N_{k}\right)$. Then there is a permutation $\rho$ in $S_{n}$ such that $\rho\left(N_{k}\right)=N_{k}, k=1, \ldots, n$ and $\sigma=\rho \pi$. It follows that $P_{\sigma}=P_{p} P_{\pi}$ where $P_{n} \in Q$. Further, $P_{p}$ is a direct sum of permutation matrices on $E_{i}$, each of which is unitary on $E_{i}$. Hence $P_{\rho} \in \mathscr{U}$. Thus $P_{\pi}=P_{\rho}^{-1} P_{o}$ is an isometry whence $P_{n} \in \mathscr{P}$.

\section{7}

THEOREM Let $v$ be a standardized absolute norm on $C_{n}$, and let $V \in C_{n n}$ be an isometry on $C_{n}$. Then there exist unique $U \in \mathscr{U}$ and $P \in \mathscr{P}$ such that $V=U P$.

Proof Let $D^{(i)}, i=1, \ldots, n$ be the diagonal matrix with $d_{i i}=1, d_{k k}=0$ for $k \neq i$. Let $K^{(i)}=V D^{(i)} V^{-1}, i=1, \ldots, n$. Since $D^{(i)} \in \mathscr{H}$, also $K^{(i)} \in \mathscr{H}$, $i=1, \ldots, n$, by (7.2). Hence $K^{(i)}=K_{1}^{(1)} \oplus \cdots \oplus K_{r}^{(i)}$, where $K_{k}^{(i)}$ is Hermitian on $E_{k}$. But $D^{(1)}, \ldots, D^{(n)}$ commute in pairs, hence so do $K^{(1)}, \ldots, K^{(n)}$. Thus there exist unitary matrices $W_{k}$ on $E_{k}$ such that $W_{k} K_{k}^{(i)} W_{k}^{-1}$ is a real diagonal matrix. Set $W=W_{1} \oplus \cdots \oplus W_{k}$. Then $W \in \mathcal{U}$, and

$$
G^{(t)}=W K^{(l)} W^{-1}=W V D^{(1)} V^{-1} W^{-1}
$$

is a real diagonal matrix for $i=1, \ldots, n$. But the $G^{(1)}$, like the $D^{(1)}$, are projections summing to $I$, and $G^{(i)} G^{(j)}=0$, for $i \neq j$. Hence $G^{(i)}=D^{\left(\sigma^{-1}(i)\right)}$, $i=1, \ldots, n$, for some permutation $\sigma$ of $\{1, \ldots, n\}$, and so $G^{(t)}=P_{\sigma}^{-1} D^{(t)} P_{\sigma}$. 
Put

$$
X=W V P_{\sigma} .
$$

Then $G^{(1)}=W V D^{(b)} V^{-1}\left(V^{-1}=X^{-1} G^{(1)} X\right.$, for $i=1, \ldots, n$. We may now deduce that $X$ is diagonal, say $X=\operatorname{ding}\left(x_{1}, \ldots, x_{n}\right)$. Hence

$$
W V e^{\sigma(l)}=X P_{\sigma}^{-1} e^{\sigma(l)}=X e^{1}=x_{1} e^{\downarrow}, \quad i=1, \ldots, n
$$

Since $W V$ is an isometry, it follows that

$$
1=v\left(W V e^{a(l)}\right)=v\left(x_{l} c^{i}\right)=\left|x_{i}\right| v\left(e^{t}\right)=\left|x_{d}\right|, \quad i=1, \ldots, n .
$$

Thus $X \in \mathscr{U}$. We now obtain that $V=W^{-1} X P_{d}^{-1}$. By (7.6), $P_{d}^{-1}=P_{d}^{-1}=$ $P_{\rho} P$, where $P_{\rho} \in \mathscr{U}, P \in \mathscr{P}$. Let $U=W^{-1} X P_{\rho}$. Then $U \in \mathscr{U}$, and $V=U P$.

To prove uniqueness, suppose that $V=U P=U^{\prime} P^{\prime}$, where also $U^{\prime} \in \mathscr{U}$, $P^{\prime} \in \mathscr{P}$, then $A=U^{\prime-1} U=P^{\prime} P^{-1} \in \mathscr{U} \cap \mathscr{P}$. But $A$ is then block permutation matrix, say $A=P_{k}$, witls $\varepsilon\left(N_{k}\right)=N_{k}, k=1, \ldots, r$. Hence $\varepsilon$ is the identity permutation and $A=I$. Tlus $U^{\prime}=U, P^{\prime}=P$ and the decomposition is unique. The theorem is proved.

Let a be a group, $b$ and it subgroups of $n$ with $n$ normal in $a$. If $n \cap b=\{1)$ and $n b=a$, then $a$ is called a semi-direct product of $n$ and $b$.

\section{8}

CoROLlary $\mathscr{V}$ is a semi-direct product $\mathscr{U}$ and $\mathscr{P}$ and $\mathscr{V} / \mathscr{U} \cong \mathscr{P}$.

Proof Sinee $\mathscr{U} \mathscr{P}=\mathscr{V}$, and $\mathscr{U}$ is normal in $\mathscr{V}$, the results are immediate by $(7.7)$.

Comment It is also clear that the connected components of $\mathscr{Y}$ are precisely the sets $\mathscr{U} P$, for $P \in \mathscr{P}$.

Remark Similarly, every $V \in \mathscr{F}$ can be represented uniquely as $V=P^{\prime} U^{\prime}$, where $P^{\prime} \in \mathscr{P}$ and $U^{\prime} \in \mathscr{U}$. Indeed, if $P^{\prime} U^{\prime}=V=U P$, then $P\left(P^{-1} U P\right)=V$, and $P^{-1} U P \in \mathscr{U}$. Hence $P^{\prime}=P$ and $U^{\prime}=P^{-1} U P$.

\section{9}

Examples 1) if $v$ is an $l_{p}$-norm, $\nu(x)=\left(\sum_{i=1}^{n}\left|x_{i}\right|^{p}\right)^{1 / p}$ where $p \geqslant 1$ and $p \neq 2$, then the equivalence classes for $\sim$ are singletons. Hence $\mathscr{U}$ consists of all diagonal matrices $U=\operatorname{diag}\left(u_{1}, \ldots, u_{n}\right)$, with $\left|u_{i}\right|=1, i=1, \ldots, n$, The group $\mathscr{P}$ consists of all permutation matrices.

2) Let $v$ be any standardized absolute norm on $C_{2}$ and suppose there is a $z \in C_{2}$ with $v\left(z_{1}, z_{2}\right) \neq 1\left(z_{2}, z_{1}\right)$. Then $\mathscr{U}$ consists of all diagonal matrices $U=\operatorname{diag}\left(u_{1}, u_{2}\right)$ with $\left|u_{1}\right|=\left|u_{2}\right|=1$, and $\mathscr{P}$ of the identity matrix. Hence $\mathscr{r}=\mathscr{U}$. 
In this section we shall restate our main results for an absolute norm $v_{1}$ on $C_{n}$ which is not necessarily standardized by $v_{1}\left(e^{t}\right)=1, i=1, \ldots, n$. Theorem $(6.2)^{\prime}$ will correspond to Theorem (6.2), etc.

Let $v_{1}\left(e^{i}\right)=d_{i}, i=1, \ldots, n$, and let $D=\operatorname{diag}\left(d_{1}, \ldots, d_{n}\right)$. Define $v(x)=v_{1}\left(D^{-1} x\right)$, for all $x \in C_{n}$. Then $v$ is a standardized absolute norm. Now define the equivalence relation $\sim$ in terms of $v$, and let $N_{l}$ and $E_{l}$ be as before. Explicitly, we now have:

and

$$
\begin{gathered}
i \sim j \quad \text { if and only if for } x, y \in C_{n}, \\
d_{i}^{2}\left|x_{i}\right|^{2}+d_{j}^{2}\left|x_{j}\right|^{2}=d_{i}^{2}\left|y_{i}\right|^{2}+d_{j}^{2}\left|y_{j}\right|^{2},
\end{gathered}
$$

imply that $v_{1}(x)=v_{1}(y)$.

Define $V(A), \mathscr{H}, \mathscr{J}, \mathscr{U}, \mathscr{V}$ as before for the standardized norm $v$, and let $V_{1}(A), \mathscr{H}_{1}, \mathscr{J}_{1}, \mathscr{U}_{1}, \mathscr{V}_{1}$ be defined correspondingly for $v_{1}$. The basic results translating theorems for $v$ into theorems for $v_{1}$ are that $v_{1}^{0}(A)=v^{0}\left(D A D^{-1}\right)$ and $\mathscr{V}_{1}(A)=V\left(D A D^{-1}\right)$ (Nirschl and Schneider [7]). Hence $K \in \mathscr{H}_{1}$ if and only if $D K D^{-1} \in \mathscr{H}$. Thus $\mathscr{H}_{1}=D^{-1} \mathscr{H} D$. Explicitly:

\section{2'}

THEOREM Let $v$ be an absolute norm on $C_{n}$, and suppose that $v\left(e^{i}\right)=d_{i}$, $i=1, \ldots, n$. Let $D=\operatorname{diag}\left(d_{1}, \ldots, d_{n}\right)$. Then $K \in C_{n n}$ is norm-Hermitian if and only if $D K D^{-1}$ is Hermition and $k_{1 j}=0$ if $i \sim j$.

Theorems (6.5) and (6.7) become

(6.5)' Let $\chi_{1}(x)=\chi(D x)=\left(\Sigma d_{i}^{2}\left|x_{i}\right|^{2}\right)^{\frac{1}{2}}$, for $x \in C_{n}$. Then, for all $A \in \mathscr{J}$, $\nu_{1}^{0}(A)=\chi_{1}^{0}(A)=\left(\rho\left(D A D^{-2} A^{*} D\right)\right)^{\frac{1}{2}}$.

(6.7) If $A$ is $v_{1}$-normal, then $D A D^{-1}$ is normal, and

$$
v_{1}^{0}(A)=\chi_{1}^{0}(A)=v_{1}(A)=\rho(A),
$$

where $v_{1}(A)$ is the numerical radius for $v_{1}$.

Finally, $\mathscr{U}_{1}=\exp \left(\mathscr{H}_{1}\right)=D^{-1} \mathscr{U} D$, and

(7.8)' The group of all isometries $\mathscr{F}_{1}$ is a semidirect product of $\mathscr{U}_{1}$ and $\mathscr{P}_{1}$, where $\mathscr{P}_{1}=D^{-1} \mathscr{P} D$ is finite.

\section{9}

In [11], Tam presents several results which, restricted to $C_{n}$, are the special cases of some of our results when the norm is invariant under every 
permutation matrix. $\dagger$ We shall show that it is possible to obtain the conclusions of [11], Theorems 2 and 3 (restricted to $C_{n}$ ) under a somewhat weaker hypothesis.

Let $G$ be a subgroup of $S_{n}$. Then $G$ is called doubly-transitive if for all ordered pairs $(i, j), i \neq j$, and all ordered pairs $(k, l), k \neq l,\{i, j, k, l\} \subseteq$ $\{1, \ldots, n\}$, there exists a permutation $\sigma \in G$ such that $\sigma(i)=k$ and $\sigma(j)=l$.

\section{1}

THEOREM Let $v$ be an absolute norm on $C_{n}$ with $v\left(e^{1}\right)=1$, and let $G$ be the subgroup of $S_{n}$ defined by $\sigma \in G$, if $P_{\sigma}$ is an isometry. If $G$ is doubly transitive, then either

a) $v=\chi$, or

b) i) $\mathscr{H}$ consists of all real diagonal matrices and, for $H \in \mathscr{H}$,

$$
v^{\circ}(H)=\max \left\{\left|h_{i i}\right|: i=1, \ldots, n\right\} .
$$

ii) $\mathscr{U}$ consists of all diagonal matrices $U$ with $\left|u_{i}\right|=1, i=1, \ldots, n$.

iii) $\mathscr{P}$ consists of all $P_{\sigma}, \sigma \in G$.

Proof Since $v\left(e^{1}\right)=1$ and $G$ is (doubly) transitive, it follows that $v\left(e^{\prime}\right)=\left(P_{\sigma} e^{1}\right)$, for suitable $\sigma \in G, i=1, \ldots, n$. Hence $v$ is standardized.

Case (a) There exist distinct $i, j$ in $\{1, \ldots, n\}$ such that $i \sim j$.

By (7.5) and the double-transitivity of $G, k \sim l$ for all $k, l$, with $k \neq l$, and $k, l \in\{1, \ldots, n\}$. Hence, by $(2.3), v=\chi$.

Case (b) Suppose all equivalence classes for $\sim$ are singletons. Then (i) follows from (6.2) and (6.5) and (ii) from (7.3). For (iii), observe that every permutation matrix which is an isometry is a block isometry.

\section{2}

Example Let $n \geqslant 3$ and $1=a_{1}>a_{2}>\cdots>a_{n} \geqslant 0$. In $C_{n}$, set

$$
\mu(x)=\sum_{i=1}^{n} a_{i}\left|x_{l}\right| \text { and } v(x)=\sup \left\{\mu\left(P_{\sigma} x\right): \sigma \in A_{n}\right\},
$$

where $A_{n}$ is the alternating group on $\{1, \ldots, n\}$. (If $n \geqslant 4$, then $A_{n}$ is doubly transitive.) Then $v$ is a standardized absolute norm on $C_{n}$, and if $G$ is defined as in Theorem (9.1), then $A_{n} \subseteq G$. Let $x=\left(a_{1}, \ldots, a_{n}\right), z=\left(a_{2}, a_{1}, a_{3}, \ldots, a_{n}\right)$. Then by a result found in Hardy, Littlewood and Polya ("Inequalities," (10.2)), it follows that $v(x)=\sum_{i=1}^{n} a_{i}^{2}>v(z)$. Hence $G \neq S_{n}$, and so $G=A_{n}$.

† We are indebted to John Duncan for pointing this out to us. This section was written after the rest of this paper was completed. 


\section{References}

[1] F. L. Bauer, On the field of values subordinate to a norm, Numer. Math. 4 (1962); 103-113.

[2] F. L. Bauer, J. Stoer, and C. Witzgall, Absolute and Monotonic Norms, Numer. Math. 3 (1961), 257-264.

[3] F. F. Bonsall and J. Duncan, Numerical Ranges of Operators on Normed Spaces, and of Elements of Normed Algebras, London Math. Soc. Lecture Note Series 2 (1971).

[4] M. J. Crabb, Some results on the numerical range of an operator, J. London Math. Soc. (2) 2 (1970), 741-745.

[5] D. Gries, Characterizations of certain classes of norms, Numer. Math. 10 (1967), 30-41.

[6] A Lumer, Semi-inner product spaces, Trans. Amer. Math. Soc. 100 (1961), $29-43$.

[7] N. Nirschl and H. Schneider, The Bauer Fields of Values of a Matrix, Numer. Math. 6(1964), 355-364.

[8] T. W. Palmer, Unbounded operators on Banach spaces, Trans. American Math. Soc. 133 (1968), 385-414.

[9] T. W. Palmer, Characterizations of $C^{*}$-algebras, Bull. American Math. Soc. 74 (1968), 538-540.

[10] I. Vidav, Eine metrische Kennzeichnung der selbstadjungierten, Operatoren. Math. Zeit. 66(1956), 121-128.

[11] K. W. Tam, Isometries of certain function spaces, Pacific J. Math. 31 (1969), 233-246.

[12] C. Zenger, On the convexity properties of the Bauer field of values of a matrix, Numer. Math, 12 (1968), 96-105.

[13] A. M. Ostrowski, Über Normen von Matrizen, Math. Zeitschrift 63 (1955), 2-18.

[14] J. Stoer and C. Witzgall, Transformations by diagonal matrices in a normal space, Numer._Math. 4 (1962), 158-171. 